\title{
Análisis biomecánico del ejercicio sentadilla libre en sujetos sin acondicionamiento físico
}

Fecha de recepción: 19 de septiembre de 2018

Fecha de aprobación: 10 de diciembre de 2018

\author{
Cristian Felipe Blanco-Díaz \\ Universidad Antonio Nariño \\ cblanco88@uan.edu.co \\ Angie Katerine Quitian-González \\ Universidad Antonio Nariño \\ aquitian119@uan.edu.co
}

DOI: https://doi.org/10.21158/23823399.v6.n0.2018.2423

Cómo citar este artículo: Blanco-Díaz, C. F.; Quitian-González, A. K. (2018). Análisis biomecánico del ejercicio sentadilla libre en sujetos sin acondicionamiento físico. Revista Ontare, 6, 59-82. DOI: https://doi.org/10.21158/23823399.v6.n0.2018.2423

\section{Resumen}

Los análisis biomecánicos proporcionan una fuente extensa de datos que son explorados ampliamente desde el punto de vista mecánico y fisiológico; dentro de estos datos se incluyen parámetros cinéticos y cinemáticos que son útiles para comprender la locomoción humana.

Por otro lado, la sentadilla es un ejercicio ampliamente utilizado para el acondicionamiento físico, ya que pone en funcionamiento al mismo tiempo varios músculos tanto del tren superior como del inferior, no obstante, una mala posición podría conducir a lesiones musculares y articulares, específicamente a nivel de la espalda y la

1 Estudiante del programa de Ingeniería Biomédica en la Universidad Antonio Nariño. ORCID: https://orcid.org/0000-0002-9877-1211

2 Estudiante del programa de Ingeniería Biomédica en la Universidad Antonio Nariño. ORCID: https://orcid.org/0000-0002-8083-7454 
rodilla, las cuales son especialmente comunes en pacientes sin acondicionamiento físico.

En este estudio, los datos de sentadillas se analizan en pacientes sin acondicionamiento físico, organizados en dos grupos, uno compuesto por mujeres y otro por hombres, para evaluar posibles implicaciones en la articulación de la rodilla.

Con la realización de este estudio se determinó que el promedio de flexión de la articulación de la rodilla para hombres y mujeres cumple con los criterios de minimización debido a que sobrepasan el grado fundamental que radica hasta los 40 grados, por lo que se pudo comprobar que aparentemente ninguna persona sufre de alguna patología en la articulación de la rodilla. Además, se pudo observar que la ausencia de ejercicio puede afectar negativamente la movilidad de las articulaciones y en el mismo sentido se evidenció cómo la amplitud articular y el desplazamiento de los segmentos se ven afectados por la edad y el peso al momento de realizar cierta clase de movimiento.

\section{Palabras clave}

Biomecánica; análisis biomecánico; datos mecánicos; datos fisiológicos; parámetros cinéticos; parámetros cinemáticos; locomoción humana. 


\title{
Biomechanical analyzes of the exercise of free squat in patients without physical conditioning
}

\begin{abstract}
Biomechanical analyzes provide an extensive source of data that are widely explored from a mechanical and physiological point of view; this data includes kinetic and kinematic parameters that are useful for understanding human locomotion.

On the other hand, the squat is an exercise that is widely used for physical conditioning, as it puts several muscles of both the upper and lower body into operation at the same time, however, a bad position could lead to muscle and joint injuries, specifically in the back and the knee, which are especially common in patients without physical conditioning.

In this study, the data from the squats are analyzed in patients without physical conditioning, organized into two groups, one composed of women and the other one composed of men, in order to assess possible implications on the knee joint.

As a result of this study, it was determined that the average flexion of the knee joint for men and women meets the criteria of minimization as they exceed the fundamental degree that lies up to 40 degrees, so it was possible to verify that apparently no person suffers from any pathology in the knee joint. In addition, it was observed that the absence of physical exercise can negatively affect the mobility of the joints, and in the same sense, it was demonstrated how the joint range and the displacement of the segments are affected by age and weight at the time of performing a certain type of movement.
\end{abstract}

\section{Keywords}

Biomechanics; biomechanical analysis; mechanical data; physiological data; kinetic parameters; kinematic parameters; human locomotion. 


\title{
Análise biomecânica do exercício de agachamento livre em indivíduos sem condicionamento físico
}

\begin{abstract}
Resumo
As análises biomecânicas fornecem uma extensa fonte de dados que é amplamente explorada do ponto de vista mecânico e fisiológico; dentro desses dados incluem parâmetros cinéticos e cinemáticos úteis para entender a locomoção humana.

Por outro lado, o agachamento é um exercício amplamente utilizado para condicionamento físico, pois coloca em operação ao mesmo tempo vários músculos da parte superior e inferior do corpo, no entanto, uma má posição pode levar a lesões musculares e articulares, especificamente nas costas e joelhos, que são especialmente comuns em pacientes sem condicionamento físico.

Neste estudo, os dados de agachamento são analisados em pacientes sem condicionamento físico, organizados em dois grupos, um composto por mulheres e outro por homens, para avaliar possíveis implicações na articulação do joelho.

Com a realização deste estudo, determinou-se que a flexão média da articulação do joelho para homens e mulheres atende aos critérios de minimização, porque excedem o grau fundamental que é de até 40 graus, portanto, verificou-se que aparentemente, nenhuma pessoa sofre de qualquer patologia na articulação do joelho. Além disso, observou-se que a ausência de exercício pode afetar negativamente a mobilidade das articulações e, no mesmo sentido, demonstrouse como a amplitude da articulação e o deslocamento dos segmentos são afetados pela idade e peso no momento da realização de um determinado tipo de movimento.
\end{abstract}

\section{Palavras-chave}

Biomecânica; análise biomecânica; dados mecânicos; dados fisiológicos; parâmetros cinéticos; parâmetros cinemáticos; locomoção humana. 


\section{Analyse biomécanique d'exercices de squat chez des personnes non entrainées}

\section{Résumé}

Les analyses biomécaniques fournissent une base de données importante $d u$ fonctionnement mécanique et physiologique largement exploité incluant des paramètres cinétiques et cinématiques utiles à la compréhension du déplacement de l'être humain.

Le squat, exercice pratiqué pour le conditionnement physique, sollicite simultanément plusieurs muscles des parties supérieures et inférieures $d u$ corps. Une mauvaise position lors des exercices peut entraîner des blessures musculaires et articulaires - en particulier au niveau du dos et des genoux particulièrement fréquentes chez les patients non entrainés.

Cette étude analyse les données de séances de squat, dont les participants sont répartis en deux groupes - l'un composé de femmes et l'autre d'hommes - afin d'évaluer les éventuelles implications sur l'articulation du genou chez ces patients sans préparation physique particulière.

L'étude conclue que la flexion moyenne du genou, chez les hommes comme chez les femmes de l'échantillon, répond aux critères de minimisation dépassant l'angle de base, d'un maximum de 40 degrés, et qu'aucun des participants ne souffrait d'une pathologie de l'articulation du genou. L'étude révèle que l'absence d'exercice pouvait avoir une incidence négative sur la mobilité des articulations et, dans le même ordre d'idée, que l'amplitude articulatoire et le déplacement des segments seront affectés par l'âge et le poids corporel.

\section{Mots-clés}

Biomécanique; analyse biomécanique; données mécaniques; données physiologiques; paramètres cinétiques; paramètres cinématiques; locomotion humaine. 


\section{Introducción}

$\mathbf{L}$

os análisis biomecánicos son extensamente utilizados debido a que permiten conocer, entender y describir de forma cuantitativa diferentes fenómenos cinéticos y cinemáticos asociados al movimiento humano.

En el caso de las sentadillas libres, se trata de un ejercicio ampliamente utilizado en sesiones de acondicionamiento físico debido a que su ejecución representa una solicitación de la mayor parte del sistema musculoesquelético.

La sentadilla se ha convertido en un objeto de estudio en áreas tales como la biomecánica, la artrología y las ciencias deportivas, entre otros. Todo esto debido a que este movimiento involucra músculos de la parte inferior y articulaciones fundamentales como, por ejemplo, la cadera, las rodillas y los tobillos, las cuales tienen cierta carga importante del peso del cuerpo. Además, la sentadilla, de manera inconsciente, está presente en muchos movimientos de la vida cotidiana de los seres humanos: en el momento de sentarse en el trabajo, en el transporte público, al recoger un objeto del suelo, etc.

Sin embargo, existen pugnas entre entrenadores, kinesiólogos y traumatólogos en las que se discuten los efectos producidos por este ejercicio, ya que para los entrenadores es uno de los ejercicios más completos y no debe causar ningún daño cuando se ejecuta con una buena técnica; no obstante, traumatólogos, kinesiólogos y profesionales de la salud indican que hay un riesgo potencial de provocar lesiones a nivel de la espalda baja y rodillas (Pereira, Corradi y Laorato, 2014).

Estudios recientes han demostrado que la buena ejecución de la sentadilla es indispensable para evitar el riesgo de lesiones a nivel de la rodilla y la espalda (Lavorato y Pereira, 2009). Sin embargo, muchas 
personas, en especial aquellas que comienzan sesiones de acondicionamiento físico o que son autodidactas, realizan dicho movimiento sin la supervisión adecuada, lo cual puede derivar en el incremento del riesgo de incurrir en una lesión osteomuscular.

La rodilla es una articulación que, durante la marcha, en una persona sana tiene una carga aproximada de $62 \%$ en el lado medial, y de un $38 \%$ en el lado lateral durante una fase de la marcha. No obstante, cuando esta presenta algún daño o patología - como, por ejemplo, valgo - la carga del compartimento medial puede aproximarse a un $100 \%$, lo cual afecta la vida cotidiana de la persona (Self, Greenwald y Pflaste, 2000).

Frente a los estudios que se han realizado sobre el efecto que produce la sentadilla en las rodillas, algunos recomiendan realizar la media sentadilla por sobre la sentadilla profunda, ya que esta última tiene mayor probabilidad de lesiones en los meniscos, así como en los ligamentos cruzados y laterales (Pereira et al., 2014).

En cuanto a la espalda baja, la controversia se centra en la compresión que genera la sobrecarga en la columna lumbar - en especial en L5 y S1-, ya que una sobrecarga excesiva puede producir una lesión con un solo movimiento, como, por ejemplo, una hernia de disco, o con movimientos sucesivos una lumbalgia mecánica; en una sentadilla con peso, una sobrecarga de $350 \mathrm{~kg}$ se considera seguro, y entre $350 \mathrm{~kg}$ y $800 \mathrm{~kg}$ generan lumbalgias, razón por la cual también es importante mantener alineada la espalda (Lavorato y Pereira, 2009; Pereira et al., 2014).

Debido a estos posibles efectos que puede tener la mala ejecución de este movimiento, se recomienda realizar la sentadilla de modo tal que una de sus piernas mantenga el pie lastrado, y obtenga así los mismos resultados musculares que una sentadilla normal, pero sin sobrecargar la columna vertebral (Lavorato y Pereira, 2009; Pereira et al., 2014). 
El análisis biomecánico de este tipo de movimientos se puede realizar por medio de diferentes métodos. Entre las técnicas que prevalecen en la lista se encuentran la videogrametría, la electromiografía (EMG), la dinamometría y las plataformas de fuerza, entre otras; cada una de estas técnicas puede sincronizarse y complementar la otra a fin de obtener mejores y más detallados resultados (Lavorato y Pereira, 2009; Nunes, Moreira y Tavares, 2016; Pereira et al., 2014; Self et al., 2000; VázquezGuerrero y Moras, 2015; Villa Moreno, Gutiérrez Gutiérrez y Pérez Moreno, 2008).

El análisis biomecánico del movimiento juega un papel importante en el manejo clínico de las afecciones neurológicas y ortopédicas en las que se realizan estudios cinemáticos y cinéticos. Por esta razón, ha existido un interés en realizar el análisis de movimiento en tiempo real y proporcionar información de manera inmediata, con el propósito de favorecer tanto al terapeuta como al paciente. Tradicionalmente, el informe del análisis de movimientos clínicos no se produce de manera inmediata, y es necesario para la toma de decisiones en el tratamiento que proporciona el médico; la información clínicamente relevante incluye variables biomecánicas tales como ángulos articulares — cinemática - y momentos articulares — cinética- (Van den Bogert, Geijtenbeek, Even-Zohar, Steenbrink y Hardin, 2013).

En este trabajo se reportan los resultados de un diseño experimental que busca evaluar el comportamiento de la articulación de la rodilla en sujetos - hombres y mujeres - sin acondicionamiento físico. El proceso de análisis biomecánico del ejercicio sentadilla libre se realizó en una muestra de diecisiete personas sin acondicionamiento físico - nueve mujeres y ocho hombres-. Se reportan variables cinemáticas como, por ejemplo, posición, velocidad y aceleración, y se distinguen tres factores que afectan las articulaciones de miembro inferior - peso, estatura y edad- A partir de los resultados obtenidos es posible concluir la importancia de la actividad deportiva en la vida diaria, en el propósito de man- 
tener el correcto funcionamiento de dichas articulaciones y reducir las posibilidades de lesiones en un futuro.

\section{Materiales y métodos}

\subsection{Sujetos de prueba}

Para este estudio se vincularon de forma voluntaria nueve mujeres y ocho hombres de mediana edad; el criterio de inclusión fue la falta de acondicionamiento físico. Inicialmente, se realiza la medición de las tres características físicas de interés en la muestra poblacional. Estás son peso, estatura y edad. Los resultados de los parámetros y medidas para cada uno de los sujetos femeninos se presentan en la tabla 1 , y los sujetos de prueba masculinos en la tabla 2.

Tabla 1. Características físicas de los sujetos de prueba femeninos

\begin{tabular}{|c|c|c|c|}
\hline Mujeres & Peso $(\mathbf{k g})$ & Estatura $(\mathbf{c m})$ & Edad (años) \\
\hline S_F1 & 56 & 160 & 20 \\
\hline S_F2 & 57 & 161 & 26 \\
\hline S_F3 & 47 & 152 & 19 \\
\hline S_F4 & 59,4 & 166 & 19 \\
\hline S_F5 & 58,9 & 163 & 19 \\
\hline S_F6 & 49 & 164 & 21 \\
\hline S_F7 & 58 & 164 & 24 \\
\hline S_F8 & 48 & 162 & 24 \\
\hline S_F9 & 81 & 162 & 32 \\
\hline
\end{tabular}

Fuente. Elaboración propia. 
Tabla 2. Características físicas de los sujetos de prueba masculinos

\begin{tabular}{|c|c|c|c|}
\hline Hombres & Peso (kg) & Estatura (cm) & Edad (años) \\
\hline S_M1 & 53 & 166 & 19 \\
\hline S_M2 & 67 & 175 & 21 \\
\hline S_M3 & 75 & 169 & 20 \\
\hline S_M4 & 53 & 169 & 18 \\
\hline S_M5 & 62 & 178 & 21 \\
\hline S_M6 & 76 & 175 & 22 \\
\hline S_M7 & 62,2 & 178 & 24 \\
\hline S_M8 & 65 & 172 & 20 \\
\hline
\end{tabular}

Fuente. Elaboración propia.

El análisis estadístico de las características físicas de las tablas 1 y 2 genera dos medias poblacionales con desviación estándar, tabuladas en la tabla 3.

Tabla 3. Valores promedio de edad, estatura y peso para hombres y mujeres que participaron en el estudio

\begin{tabular}{|l|c|c|c|}
\hline & Edad (años) & Estatura (cm) & Peso (kg) \\
\hline Mujeres & $22,7 \pm 4,4$ & $161 \pm 4$ & $57,4 \pm 10,2$ \\
\hline Hombres & $20,6 \pm 1,8$ & $172,7 \pm 4,4$ & $64,2 \pm 8,7$ \\
\hline
\end{tabular}

Fuente. Elaboración propia.

\subsection{Diseño experimental y registro de movimientos}

Para la toma de muestras en video de la ejecución del movimiento se utilizan dos cámaras de alta velocidad Basler AG, y para la digitalización el software Maxtraq 2D y 3D - Innovision-Systems-, el cual permite distinguir la posición respecto al tiempo de marcadores reflectantes ubicados como referencia en diferentes secciones anatómicas de interés. En la construcción del modelo biomecánico y el análisis de amplitud articu- 
lar se utiliza el software Mokka Biomechanics, a partir del cual también se exportan los datos de posición para utilizarlos en el cálculo de otras variables cinemáticas por medio de funciones construidas en el software MATLAB — Campus Licence UAN-.

Para el registro se utilizaron diez marcadores pasivos — como se muestra en la Figura 1-, los cuales se ubicaron y labelizaron siguiendo las recomendaciones de la International Society of Biomechanics o ISB: en la cadera — RASI y LASI - , en las rodillas — RKNE y LKNE—, en los tobillos - RANK y LANK-, en los pies - RTOE y LTOE-, en la pelvis - PLVS - y, por último, cerca al centro de gravedad - STRN-. Además, se utiliza la denominación R para derecha y L para izquierda. Con ayuda del software MaxTraq 2D se realiza la sincronización de las cámaras y la adquisición de los movimientos para cada uno de los sujetos. También con Maxtraq 3D se digitalizan, lo que permite la visualización en la plataforma de acceso gratuito Mokka Biomechanics.

Figura 1. Simulación del movimiento ejecutado por el sujeto F2 en la plataforma Mokka

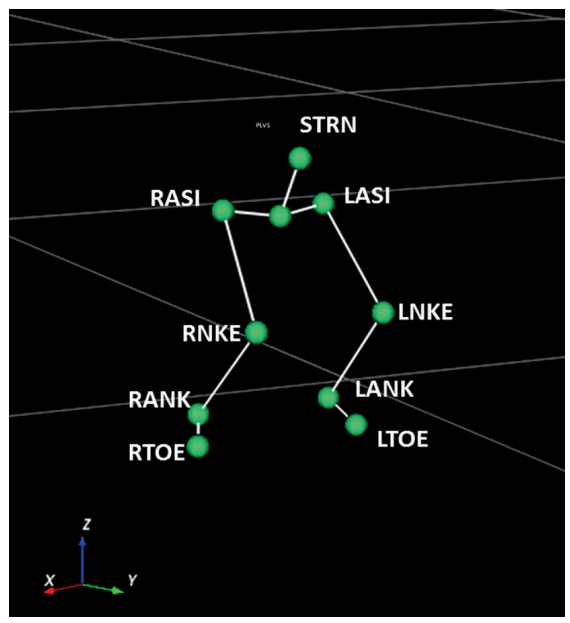

Fuente. Elaboración propia. 


\subsubsection{Análisis estadísticos.}

Los resultados obtenidos se compararon por medio del método de T-Test para evaluar si existen diferencias estadísticamente significativas $-\mathrm{p}<0,05$ - entre géneros.

\subsubsection{Consentimiento informado}

De acuerdo con las recomendaciones establecidas en la Declaración de Helsinki, los sujetos firmaron un consentimiento informado en el que expresan su participación voluntaria en el presente estudio y haber comprendido todo el procedimiento, el manejo y el aprovechamiento de los resultados.

\section{Resultados y discusión}

Figura 2. Fases del movimiento a) posición inicial; b) descenso o bajada; c) punto máximo de flexión; d) ascenso o subida

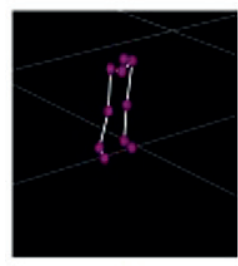

a)

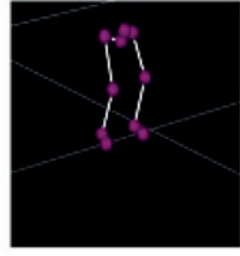

b)

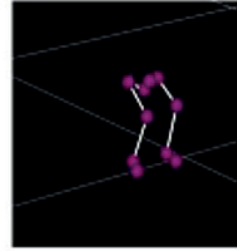

c)

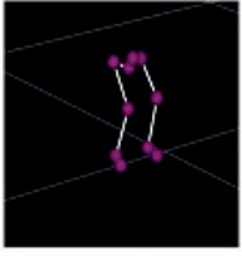

d)

Fuente. Elaboración propia.

En la Figura 2 se distinguen cuatro fases de la sentadilla que se usan de referencia como posición inicial, final y puntos críticos en el análisis.

La plataforma MATLAB tiene la capacidad de convertir los datos .txt en vectores, de modo que facilita las operaciones con estos valores. Principalmente, en este documento se hace énfasis en los marcadores de 
las rodillas, debido a que la sentadilla es la articulación con más trabajo. En un principio se pueden graficar la posición de los marcadores en los tres ejes $-\mathrm{X}, \mathrm{Y}, \mathrm{Z}$ - con respecto al tiempo.

Figura 3. Posición de los marcadores de las rodillas - M3 y M8 - con respecto al tiempo del sujeto F5

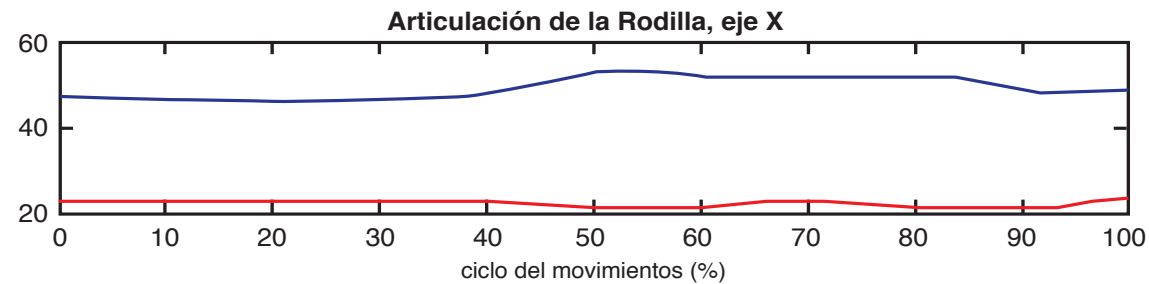

Articulación de la Rodilla, eje Y

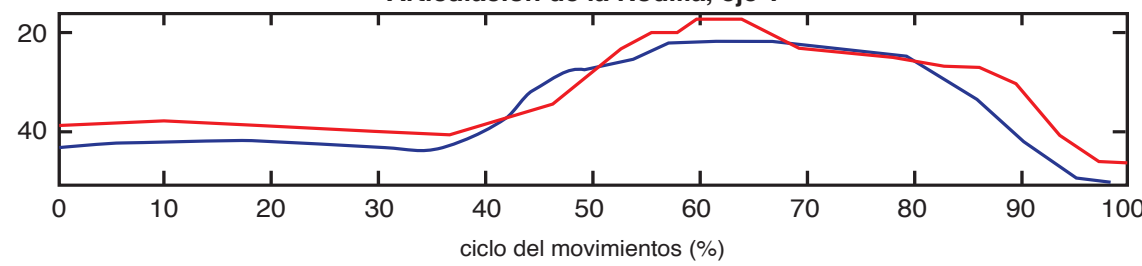

Articulación de la Rodilla, eje $X$

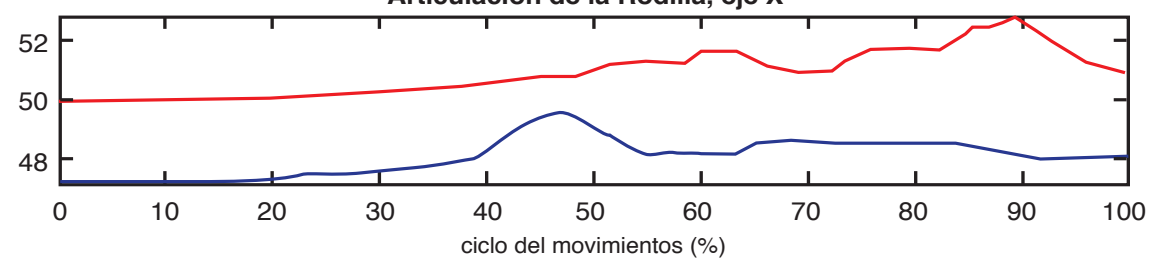

Fuente. Elaboración propia. 
Figura 4. Posición de los marcadores de las rodillas - $\mathrm{M} 3$ y M8 - con respecto al tiempo del sujeto M1

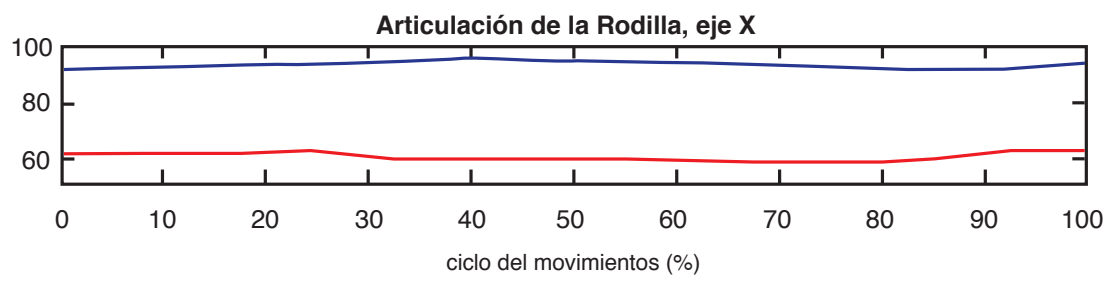

Articulación de la Rodilla, eje Y

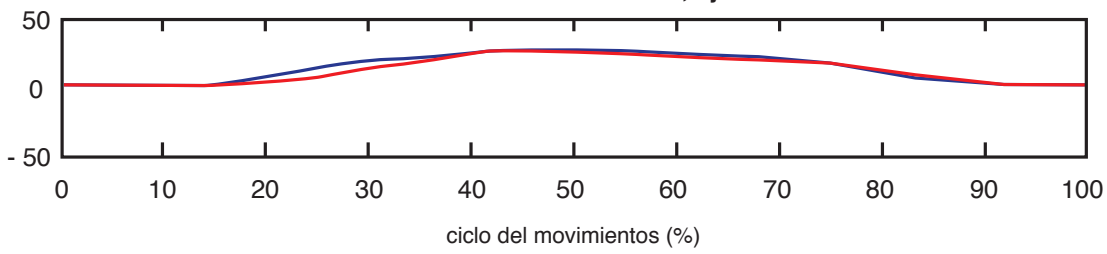

Articulación de la Rodilla, eje X

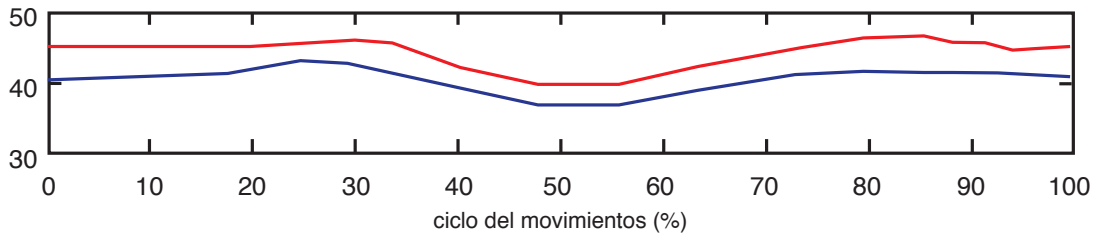

Fuente. Elaboración propia.

Como se observa en las figuras 3 y 4 , lo ideal es que los dos marcadores tengan un comportamiento parecido, por lo que en la sentadilla las dos rodillas ejecutan el movimiento coordinado. En razón a que en este estudio no se obtuvieron esos resultados, se buscó profundizar más en analizar este fenómeno, de manera que, posteriormente, se crea un código en MATLAB, lo cual permita el análisis de la amplitud articular que existe en ambas piernas, al tener en cuenta el punto central: la rodilla. Por tanto, se calcula el ángulo entre los segmentos rodilla-tobillo y rodilla-cadera, y se grafica la variación de este ángulo con respecto al tiempo. 
Figura 5. Variación del ángulo articular en cada pierna del sujeto M3 con respecto al tiempo

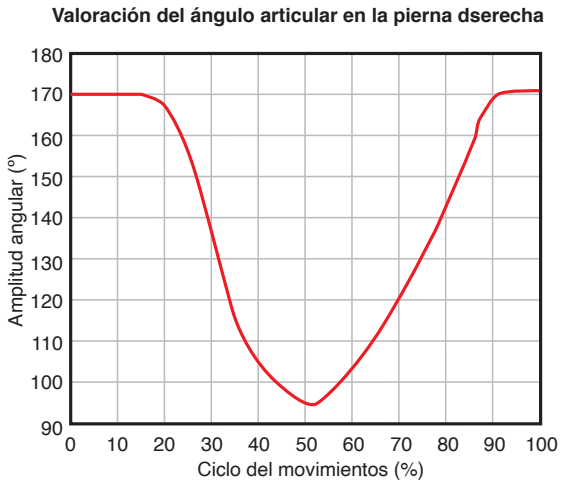

Valoración del ángulo articular en la pierna izquierda

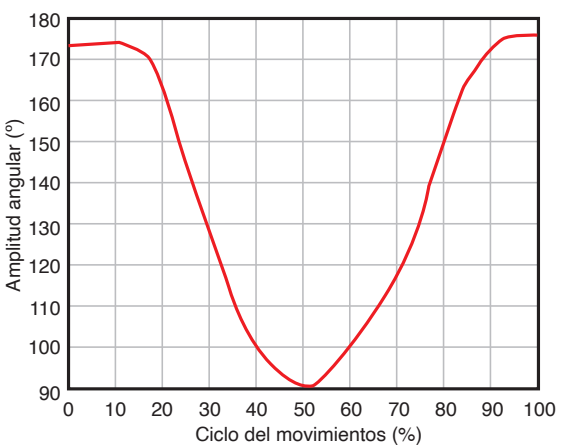

Fuente. Elaboración propia.

Figura 6. Variación del ángulo articular en cada pierna del sujeto F5 con respecto al tiempo
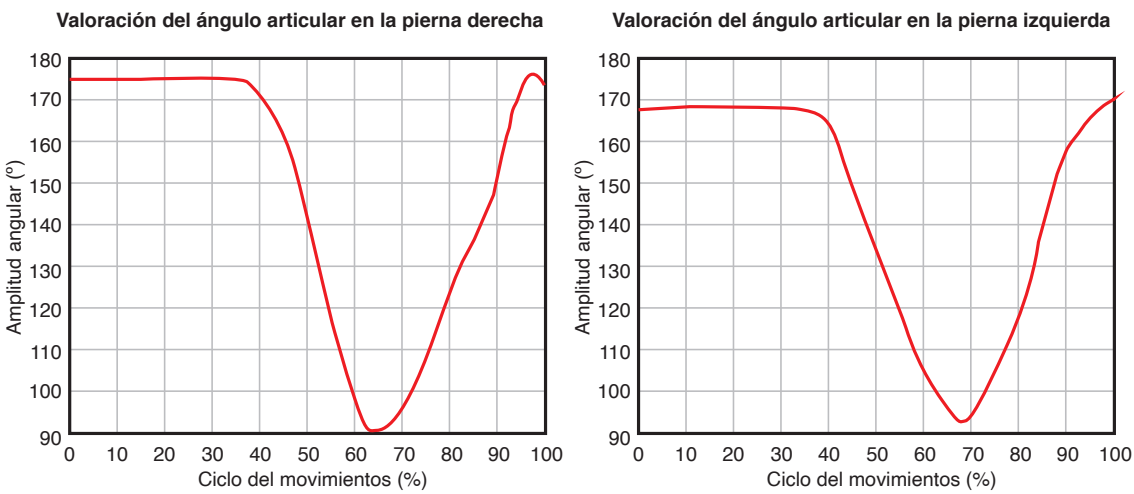

Fuente. Elaboración propia.

Como se observa en las figuras 5 y 6 , a pesar de que la amplitud articular en las dos piernas tiene comportamientos similares, existe una ligera diferencia de amplitud articular en el momento de graficar todas las muestras. Por tanto, a fin de aumentar la precisión de esta diferencia se realiza un código que permita calcular la amplitud articular de la articu- 
lación de la rodilla; además, al conocerla, así como el tiempo de ejecución del movimiento, es posible también calcular la velocidad angular y la aceleración angular.

Tabla 4. Tabla de amplitud articular, velocidad y aceleración angular de los hombres

\begin{tabular}{|c|c|c|c|c|c|}
\hline \multicolumn{6}{|c|}{ Hombres - pierna izquierda- } \\
\hline \multirow{2}{*}{ Sujeto } & \multirow{2}{*}{$\begin{array}{c}\text { Amplitud } \\
\left({ }^{\circ}\right)\end{array}$} & \multicolumn{2}{|c|}{ Velocidad angular (rad/s) } & \multicolumn{2}{|c|}{$\begin{array}{c}\text { Aceleración angular } \\
\left(\mathrm{rad} / \mathrm{s}^{2}\right)\end{array}$} \\
\hline & & Bajada & Subida & Bajada & Subida \\
\hline S_M1 & 84,64 & 1,09 & 0,76 & 0,87 & 0,39 \\
\hline S_M2 & 75,25 & 0,95 & 0,64 & 0,70 & 0,31 \\
\hline S_M3 & 84,05 & 1,00 & 0,84 & 0,68 & 0,54 \\
\hline S_M4 & 49,85 & 0,32 & 0,29 & 0,12 & 0,14 \\
\hline S_M5 & 73,82 & 0,68 & 1,65 & 0,36 & 2,12 \\
\hline S_M6 & 43,18 & 0,23 & 0,43 & 0,08 & 0,25 \\
\hline S_M7 & 80,80 & 0,88 & 0,77 & 0,69 & 0,44 \\
\hline S_M8 & 76,23 & 0,66 & 0,83 & 0,34 & 0,53 \\
\hline PROM & 70,98 & 0,72 & 0,78 & 0,48 & 0,59 \\
\hline \multicolumn{6}{|c|}{ Hombres - pierna derecha- } \\
\hline & \multirow{2}{*}{$\begin{array}{c}\text { Amplitu } \\
\left({ }^{\circ}\right)\end{array}$} & \multicolumn{2}{|c|}{ Velocidad angular (rad/s) } & \multicolumn{2}{|c|}{ Aceleración angular $\left(\mathrm{rad} / \mathrm{s}^{2}\right)$} \\
\hline & & Bajada & Subida & Bajada & Subida \\
\hline S_M1 & 90,49 & 1,26 & 0,75 & 1,08 & 0,37 \\
\hline S_M2 & 81,74 & 0,66 & 1,03 & 0,33 & 0,75 \\
\hline S_M3 & 76,89 & 0,87 & 0,88 & 0,57 & 0,60 \\
\hline S_M4 & 41,75 & 0,34 & 0,24 & 0,16 & 0,09 \\
\hline S_M5 & 62,80 & 0,55 & 1,37 & 0,29 & 1,85 \\
\hline S_M6 & 45,68 & 0,24 & 0,45 & 0,09 & 0,26 \\
\hline S_M7 & 77,88 & 0,98 & 0,78 & 0,75 & 0,45 \\
\hline S_M8 & 69,31 & 0,64 & 0,74 & 0,34 & 0,46 \\
\hline PROM & 68,32 & 0,69 & 0,78 & 0,45 & 0,60 \\
\hline
\end{tabular}

Fuente. Elaboración propia. 
Tabla 5. Tabla de amplitud articular, velocidad y aceleración angular de las mujeres

\begin{tabular}{|c|c|c|c|c|c|}
\hline \multicolumn{6}{|c|}{ Mujeres — pierna izquierda)_- } \\
\hline \multirow{2}{*}{ Sujeto } & \multirow{2}{*}{$\begin{array}{l}\text { Amplitud } \\
\left(^{\circ}\right)\end{array}$} & \multicolumn{2}{|c|}{$\begin{array}{l}\text { Velocidad angular } \\
(\mathrm{rad} / \mathrm{s})\end{array}$} & \multicolumn{2}{|c|}{$\begin{array}{c}\text { Aceleración angular } \\
\left(\mathrm{rad} / \mathrm{s}^{2}\right)\end{array}$} \\
\hline & & Bajada & Subida & Bajada & Subida \\
\hline S_F1 & 62,78 & 0,39 & 0,58 & 0,14 & 0,34 \\
\hline S_F2 & 110,29 & 1,48 & 1,71 & 1,19 & 1,53 \\
\hline S_F3 & 80,98 & 0,82 & 1,19 & 0,48 & 1,01 \\
\hline S_F4 & 89,57 & 0,64 & 0,83 & 0,27 & 0,44 \\
\hline S_F5 & 67,69 & 0,47 & 1,00 & 0,20 & 0,85 \\
\hline S_F6 & 78,56 & 1,27 & 0,95 & 1,27 & 0,70 \\
\hline S_F7 & 50,26 & 0,55 & 0,65 & 0,36 & 0,53 \\
\hline S_F8 & 61,73 & 0,48 & 0,83 & 0,21 & 0,66 \\
\hline S_F9 & 51,50 & 0,49 & 0,86 & 0,38 & 0,83 \\
\hline PROM & 72,60 & 0,73 & 0,96 & 0,50 & 0,76 \\
\hline \multicolumn{6}{|c|}{ Mujeres — pierna Derecha- } \\
\hline & & \multicolumn{2}{|c|}{$\begin{array}{c}\text { Velocidad angular } \\
\text { (rad/s) }\end{array}$} & \multicolumn{2}{|c|}{$\begin{array}{c}\text { Aceleración angular } \\
\left(\mathrm{rad} / \mathrm{s}^{2}\right)\end{array}$} \\
\hline & $\left({ }^{\circ}\right)$ & Bajada & Subida & Bajada & Subida \\
\hline S_F1 & 63,59 & 0,41 & 0,63 & 0,15 & 0,36 \\
\hline S_F2 & 112,10 & 1,40 & 1,78 & 1,11 & 1,62 \\
\hline S_F3 & 85,62 & 0,82 & 1,20 & 0,49 & 0,97 \\
\hline S_F4 & 90,59 & 0,67 & 0,83 & 0,28 & 0,44 \\
\hline S_F5 & 76,73 & 0,58 & 1,00 & 0,25 & 0,78 \\
\hline S_F6 & 80,68 & 1,21 & 0,96 & 1,32 & 0,67 \\
\hline So_F7 & 53,57 & 0,52 & 0,77 & 0,32 & 0,69 \\
\hline S_F8 & 66,17 & 0,52 & 0,88 & 0,23 & 0,70 \\
\hline S_F9 & 50,22 & 0,54 & 0,85 & 0,40 & 0,83 \\
\hline PROM & 75,47 & 0,74 & 0,99 & 0,51 & 0,78 \\
\hline
\end{tabular}

Fuente. Elaboración propia.

Como se puede observar en las tablas 4 y 5 , existen casos en los que, en los sujetos de prueba, la amplitud articular varía entre una pierna y la otra. Además, casos en los que una pierna, en el momento de descender y 
ascender, lo hace con más aceleración y rapidez en el movimiento. En el momento de comparar este tipo de resultados sobre la pierna con mayor amplitud articular, velocidad y aceleración con respecto a las características físicas de los sujetos de prueba, se descubre que estos valores fundamentan la pierna hábil del sujeto, por lo que se genera como resultado una sobrecarga hacia la pierna más hábil en la ejecución del movimiento, mas no de forma equitativa como se esperaría en sujetos con acondicionamiento físico y experiencia en ejercicios del tronco inferior tales como las sentadillas.

Además de esta tabla, también se obtienen resultados acerca de la variación de la amplitud articular, lo que demuestra cómo las personas tanto hombres como mujeres - que sobrepasan la edad media tienen, la mayoría, menor amplitud articular con relación a aquellas que se encuentran por debajo de la edad promedio. Esto fundamenta el hecho de que el ser humano, con el paso del tiempo, pierde líquido sinovial y colágeno, lo que genera una pérdida de propiedades mecánicas en las articulaciones. Lo mismo sucede con aquellas personas cuyo peso sobrepasa el peso promedio, debido a que este movimiento, al usar el peso corporal, aumenta el nivel de dificultad si el peso corporal aumenta. Además, esta amplitud también varía con respecto al género. Se obtuvo resultados en los que se puede fundamentar el hecho de que las mujeres son más flexibles con respecto a los hombres debido a que las mujeres tuvieron una amplitud articular de aproximadamente $75,6^{\circ}$ y $75,5^{\circ}$, y los hombres de $71^{\circ}$ y $68,3^{\circ}$.

Otro dato interesante radica en que, dado que las personas de este estudio no tenían acondicionamiento físico, en el momento de realizar la sentadilla el tronco inferior de la mayoría de los sujetos de pruebas tuvo dificultades en la ejecución del movimiento, por lo que estos segmentos, al ser cadenas cinéticas cerradas, tendían a inestabilizarse. Este fenómeno quedó registrado debido a que en todas las muestras existe un gran desplazamiento en el eje $\mathrm{X}$ de los marcadores de las rodillas, en el que el movimiento ideal no tendría que tener desplazamientos en este eje. 
Figura 7. Desplazamiento en el eje X de las rodillas del sujeto F2
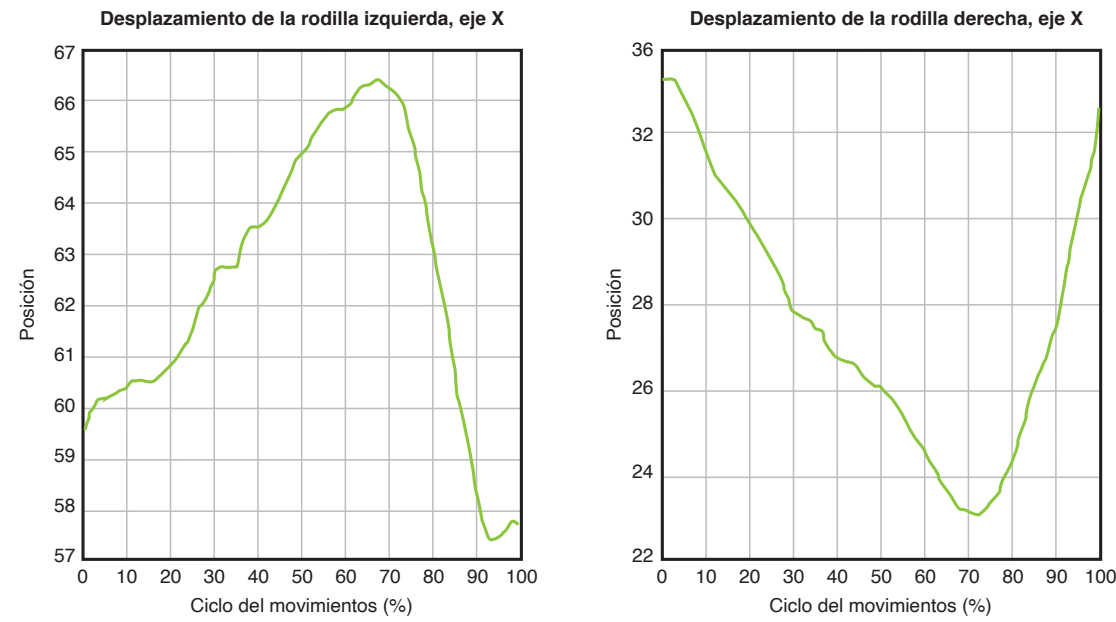

Fuente. Elaboración propia.

Figura 8. Desplazamiento en el eje $\mathrm{X}$ de las rodillas del sujeto M4
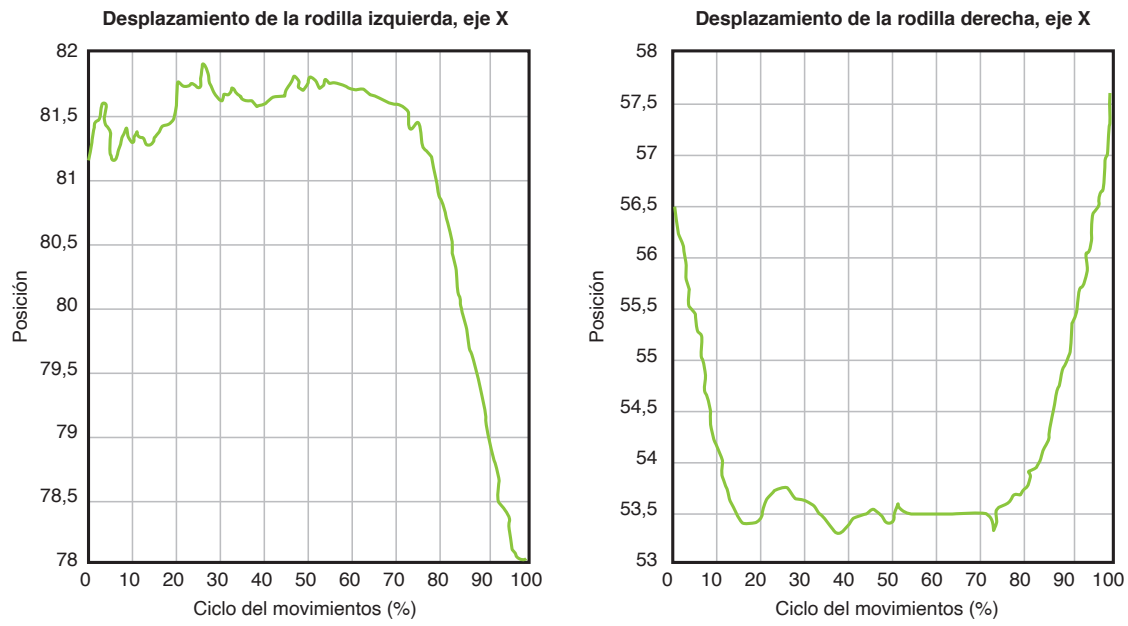

Fuente. Elaboración propia. 
El movimiento que realizan en atletas de gran rendimiento, al ser un movimiento casi ideal, conservaría un desplazamiento en el eje $\mathrm{X}$ casi nulo o nulo. En el caso de los sujetos de este estudio, al no ser deportistas de alto rendimiento y no tener un acondicionamiento físico, se puede apreciar que existe un gran desplazamiento de rodilla en el movimiento.

Las pruebas estadísticas realizadas entre los promedios de los valores obtenidos no mostraron diferencias significativas entre los géneros - p >0,05 - para la amplitud articular del movimiento. Sin embargo, en la velocidad y la aceleración durante la subida si se encontraron diferencias estadísticamente significativas entre los géneros $-\mathrm{p}<0,05-$, de modo que se puede apreciar que las mujeres tienden a subir mucho más rápido que los hombres.

Tabla 6. Desplazamiento total de las rodillas en los sujetos de prueba

\begin{tabular}{|c|c|c|c|c|c|}
\hline \multicolumn{3}{|c|}{ Mujeres } & \multicolumn{3}{|c|}{ Hombres } \\
\hline & 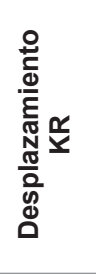 & 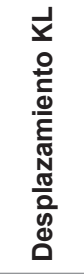 & & 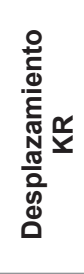 & 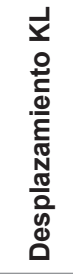 \\
\hline S_F1 & 4,55 & 3,89 & S_M1 & 9,06 & 8,86 \\
\hline S_F2 & 9,04 & 10,28 & S_M2 & 10,07 & 4,25 \\
\hline S_F3 & 8,48 & 5,37 & S_M3 & 4,16 & 4,37 \\
\hline S_F4 & 14,15 & 16,00 & S_M4 & 3,88 & 4,30 \\
\hline S_F5 & 7,50 & 3,53 & S_M5 & 8,44 & 7,83 \\
\hline
\end{tabular}




\begin{tabular}{|r|l|l|l|l|l|}
\hline S_F6 & 7,07 & 8,10 & S_M6 & 3,00 & 8,10 \\
\hline S_F7 & 6,09 & 6,27 & S_M7 & 3,81 & 5,79 \\
\hline S_F8 & 3,88 & 9,63 & S_M8 & 9,53 & 5,93 \\
\hline S_F9 & 3,14 & 5,02 & & & \\
\hline PROM & 7,10 & 7,57 & PROM & 6,49 & 6,18 \\
\hline
\end{tabular}

Fuente. Elaboración propia.

También se puede observar que en las figuras 7 y 8 la línea graficada no es totalmente suavizada, sino que conserva muchos cambios entre datos. Esto se debe a que, como el cuerpo de estos sujetos de prueba no está acostumbrado a la actividad física, casi todos temblaron en el momento de realizar la adquisición del movimiento.

\section{Conclusiones}

T primer lugar, se concluye que este tipo de análisis es muy útil para descubrir fallas o déficits en el rango de movimiento - ROM por sus siglas en inglés - de las articulaciones. Esto puede ser una herramienta de apoyo para las evaluaciones funcionales en los tratamientos de rehabilitación, en especial en la recuperación de la elasticidad o para potenciar estas estructuras morfológicas con un objetivo específico, como, por ejemplo, el acondicionamiento deportivo.

Una segunda conclusión es que a fin de obtener los beneficios que ofrece la sentadilla - tales como mejorar la musculatura de la parte inferior, mejorar el rendimiento deportivo, la movilidad y obtener una buena 
rehabilitación, entre otros-, es necesario aplicar una técnica adecuada. En caso de no haberla realizado antes es importante asesorarse acerca de esta para no causar daño a nivel muscular ni en las articulaciones.

En tercer lugar, se concluye que, con la realización de este estudio, se determinó que el promedio de flexión de la articulación de la rodilla para hombres y mujeres cumple con los criterios de minimización, debido a que sobrepasan el grado fundamental que radica hasta los 40 grados, por lo que se pudo comprobar que, cuantitativamente, ninguna persona - aparentemente - sufre de alguna patología en la articulación de la rodilla.

Una cuarta conclusión es que con este análisis fue posible observar algunas de las consecuencias de no realizar algún tipo de acondicionamiento que mantenga las articulaciones activas. La ausencia de ejercicio puede afectar de forma negativa la movilidad de las articulaciones. En los resultados presentados se evidencia cómo la amplitud articular y el desplazamiento de los segmentos se ven afectados por la edad y el peso en el momento de realizar cierta clase de movimiento.

Por último, a pesar de que los pies están ubicados de forma paralela, y que al momento de realizar sentadillas los talones no se levantan del suelo, la fuerza del peso no está administrada por igual en cada rodilla. Tal como se pudo observar en las gráficas, por medio de la posición de los marcadores, de la velocidad y de la aceleración angular hay más concentración del peso en un segmento que en el otro, lo cual se da, normalmente, en la pierna más hábil del sujeto. 


\section{Referencias}

Lavorato, M. A.; Pereira, N. V. (2009). La sentadilla, ¿es un ejercicio potencialmente lesivo? Investigación y Desarrollo, 1(1), 1-12.

Nunes, J. F.; Moreira, P. M.; Tavares, J. M. (2016). Análisis de movimiento humano y herramientas de simulación: una encuesta. En F. Miranda y C. Abreu (Eds.), Manual de investigación sobre simulación computacional y modelado en ingeniería (359-388). Hershey, PA: IGI Global. DOI: 10.4018978-1-4666-8823-0.ch012

Pereira, N.; Corradi, C.; Laborato, M. (2014). Comparación entre la sentadilla y la "sentadilla a una pierna con pie lastrado" con respecto a la actividad electromiográfica, la fuerza resultante y la sobrecarga. Revista de Entrenamiento Deportivo, 28(4). Recuperado de https://g-se.com/ comparacion-entre-la-sentadilla-y-la-sentadilla-a-una-pierna-conpie-lastrado-con-respecto-a-la-actividad-electromiografica-la-fuerzaresultante-y-la-sobrecarga-1427-sa-557cfb2720c3f2

Self, B. P.; Greenwald, R. M.; Pflaste, D. S. (2000). A biomechanical analysis of a medial unloading brace for osteoarthritis in the knee. Arthritis Care Research, 13(4), 191-197. DOI: https://doi. org/10.1002/1529-0131(200008)13:4<191::AID-ANR3>3.0.CO;2-C

Van den Bogert, A. J.; Geijtenbeek, T.; Even-Zohar, O.; Steenbrink, F.; Hardin, E. C. (2013). A real-time system for biomechanical analysis of human movement and muscle function. Medical Biological Engineering Computing, 51(10), 1069-1077. DOI: https://doi.org/10.1007/ s11517-013-1076-z

Vázquez-Guerrero, J.; Moras, G. (2015). Cambios en la arquitectura muscular y en la velocidad de ejecución de sentadillas en VersaPulley en condiciones estables e inestables en jugadores junior de baloncesto de élite durante una temporada. Cuadernos de Psicología del Deporte, 15(3), 243-252. DOI: https://doi.org/10.4321/ S1578-84232015000300029 
Villa Moreno, A.; Gutiérrez Gutiérrez, E.; Pérez Moreno, J. C. (2008). Consideraciones para el análisis de la marcha humana. Técnicas de videogrametría, electromiografía y dinamometría. Revista Ingeniería Biomédica, 2(3), 16-26 